\title{
8
}
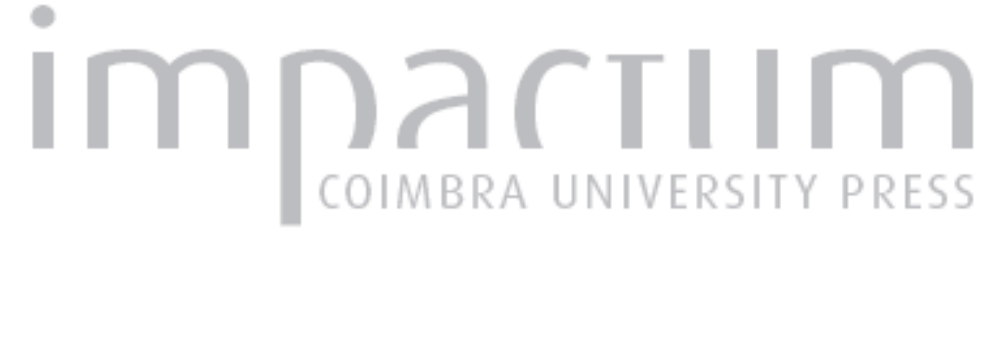

\section{Il canone verdiano in Portogallo: formazione e funzioni}

\section{Autor(es): Rostagno, Antonio}

Publicado por: Imprensa da Universidade de Coimbra

URL persistente:

URl:http://hdl.handle.net/10316.2/42427

DOI:

DOI:https://doi.org/10.14195/0870-8584_8_3

Accessed : $\quad$ 26-Apr-2023 16:15:17

A navegação consulta e descarregamento dos títulos inseridos nas Bibliotecas Digitais UC Digitalis, UC Pombalina e UC Impactum, pressupõem a aceitação plena e sem reservas dos Termos e Condições de Uso destas Bibliotecas Digitais, disponíveis em https://digitalis.uc.pt/pt-pt/termos.

Conforme exposto nos referidos Termos e Condições de Uso, o descarregamento de títulos de acesso restrito requer uma licença válida de autorização devendo o utilizador aceder ao(s) documento(s) a partir de um endereço de IP da instituição detentora da supramencionada licença.

Ao utilizador é apenas permitido o descarregamento para uso pessoal, pelo que o emprego do(s) título(s) descarregado(s) para outro fim, designadamente comercial, carece de autorização do respetivo autor ou editor da obra.

Na medida em que todas as obras da UC Digitalis se encontram protegidas pelo Código do Direito de Autor e Direitos Conexos e demais legislação aplicável, toda a cópia, parcial ou total, deste documento, nos casos em que é legalmente admitida, deverá conter ou fazer-se acompanhar por este aviso. 


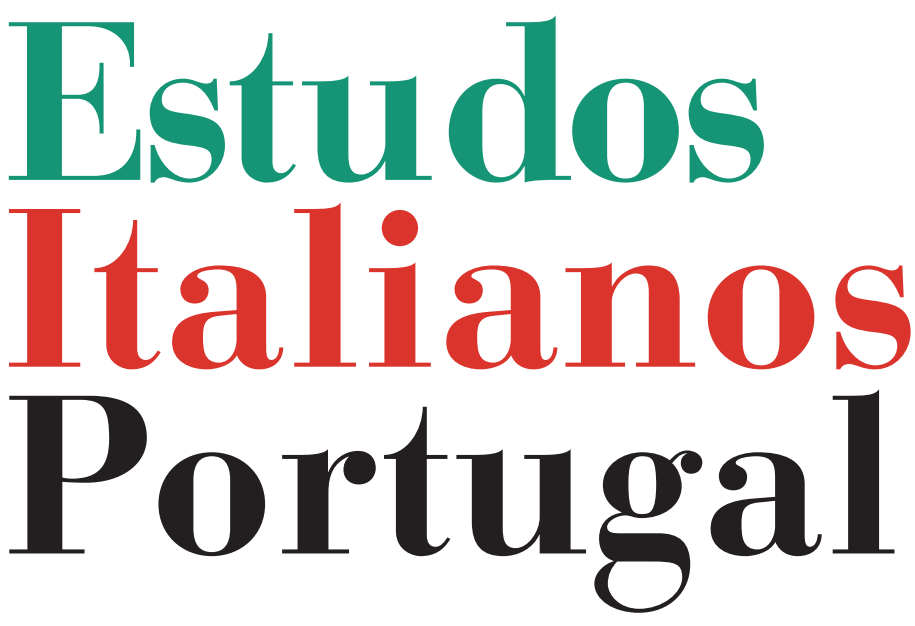

Instituto

Italiano

de Cultura

de Lisboa

Nova Série

$\mathbf{N}^{\circ} \mathbf{8}$ 


\section{IL CANONE VERDIANO IN PORTOGALLO. FORMAZIONE E FUNZIONI}

Antonio Rostagno*

Sarebbe facile trattare il tema limitandosi a dati e date. Sempre utile potrebbe essere un elenco delle prime esecuzioni nei maggiori teatri e una statistica commentata dei titoli più frequenti. Ma una sistemazione simile esiste già, e ad essa si rimanda per avere tutte le informazioni necessarie ${ }^{1}$. Il lavoro di Luísa Cymbron dimostra che i titoli più frequenti nei teatri di Lisbona e Porto non si differenziano dal repertorio verdiano che si sta affermando in tutta Europa, con la centralità via via assegnata a Nabucco, la Trilogia, Un ballo in maschera, Aida e Otello. E questi titoli sono eseguiti in Portogallo pochi mesi dopo le prime italiane, come accade più o meno in tutto il resto del mondo musicale internazionale.

\footnotetext{
* Ricercatore di Musicologia presso la Sapienza-Università di Roma, dove insegna Storia della Musica e Drammaturgia musicale. Dal 2001 collabora con l'Istituto Nazionale di Studi Verdiani di Parma per diversi progetti di ricerca: storia dei teatri in Italia, storia della direzione d'orchestra nel periodo di Verdi ecc. Sta per uscire a sua cura il Carteggio fra Verdi e Angelo Mariani, il principale direttore d'orchestra italiano dell'Ottocento. Ha scritto diversi libri dedicati al compositore tedesco Robert Schumann, fra cui: Kreisleriana di Robert Schumann (2007) e Le ultime opere di Robert Schumann: analisi dei "Gesänge der Frühe» per pianoforte op. 133 (2011).

${ }^{1}$ Luísa Cymbron, "A produção e recepção das óperas de Verdi em Portugal no século XIX”, in Verdi em Portugal (1843-2001). Catálogo da Exposição comemorativa do centenário da morte do compositor, Lisboa, Biblioteca Nacional, Teatro Nacional de São Carlos, 2001.
} 
Dunque dal punto di vista storico-musicale, il rapporto fra Verdi e il Portogallo non contiene elementi distintivi, e per questo sembrerebbe un tema poco interessante. Eppure quel rapporto fu molto lungo, e il suo esame offre elementi di riflessione utili non solo alla storia locale-nazionale ma anche alla storiografia verdiana.

La lunga relazione fra Verdi e il Portogallo inizia prestissimo. Il nome da cui partire è quello di Angelo Frondoni (1809-1891); perché? cosa lo lega a Verdi? poco, in realtà, ma quel poco non è del tutto trascurabile. Nel 1838 Frondoni fu ingaggiato dal teatro maggiore di Lisbona, il São Carlos, come maestro concertatore ${ }^{2}$. E probabile che fra gli aspiranti ad ottenere quel posto ci fosse anche il giovane Verdi, allora confinato alla periferia dell'impero, a Busseto, dopo il termine degli studi a Milano, e ansioso di tornare vicino ai centri della vita musicale. La notizia della possibile candidatura del giovane Verdi viene riportata, ma non documentata, da diversi cronisti novecenteschi, e anche Cymbron la riporta sia pur con molte cautele . $^{3}$

Questa vicenda testimonia la corrente di cultura musicale italiana verso il Portogallo, grazie alla quale trasmigrano dai centri produttivi italiani una quantità di partiture e di uomini (cantanti, strumentisti d'orchestra, compositori, impresari), per un giro d'affari notevole. Al São Carlos di Lisbona, per fare qualche esempio, sia i primi violini direttori d'orchestra, sia i maestri concertatori furono spesso italiani, nel 1838, quando arrivò Frondoni, il primo violino era Vincenzo (Vicente) Tito Mazzoni, e ancora negli anni Cinquanta, quando le due funzioni di concertare e dirigere l'orchestra vengono riunite in una sola persona, la scelta cadde ancora su professionisti italiani: dapprima Carlo Dubini, dal 1860 Pietro

${ }^{2}$ Il maestro concertatore, di solito un pianista-compositore, era colui che preparava i cantanti a pianoforte, prima di iniziare le prove con l'orchestra, e adattava le partiture alle qualità dei solisti, scrivendo le cosiddette "puntature".

${ }^{3}$ Luísa Cymbron, op. cit., p. 22. 
Antonio Coppola e dal 1863 il pianista Emilio Lami ${ }^{4}$. Forse oggi facciamo fatica a comprendere quanto ancora nell'Ottocento il melodramma costituisse il solo bene d'esportazione nel quale l'Italia fosse egemone, dall'Argentina all'Australia, da Bucarest a Lisbona.

Frondoni è una fra le tante figure che attraverso gli anni Trenta gravitavano intorno al teatro alla Scala, ottenendo ingaggi sia come compositore sia come maestro concertatore, e più o meno simile era la condizione di Verdi prima del $\mathrm{Na}-$ bucco. Come Verdi, Frondoni veniva dalla padana parmense: era nato a Zibello, quattro case vicino a Busseto, da cui proveniva anche Emanuele Muzio 5 . Come Verdi, Frondoni aveva studiato pianoforte e composizione (a Parma). E presto, come Verdi, aveva iniziato a guadagnarsi da vivere nel basso artigianato musicale, organista a Soragna dal 1829 al 1833. Ma, sempre come Verdi, la vita di provincia gli stava stretta e nel 1833 gli sforzi per uscire dal grigiore della provincia lo portarono a debuttare alla Scala di Milano con la sua prima opera comica, Il carrozzino da vendere, seguita nel 1835 dal successo della farsa Un terno al lotto al Teatro Carcano. Non sfuggirà la esatta identità con il percorso biografico del giovane Verdi, che per seguire l'Oberto conte di San Bonifacio alla Scala abbandonò definitivamente il lavoro a Busseto, con coraggio e una buona dose d'incoscienza. Ma mentre Verdi

\footnotetext{
${ }^{4}$ Manuel Carlos de Brito, Luísa Cymbron, "Opera Orchestra in the 18th and 19th Centuries in Lisbon and Oporto", in Musical Life in Europe 1600-1900. Circulation, Institutions, Representation. The Opera Orchestra in 18th- and 19th-Century Europe (I: "The Orchestra in Society" Volume 2), ed. N. M. Jensen, F. Piperno, Berlin, BWV Berliner Wissenschafts Verlag, 2007, pp. 441-475, in particolare 457-458.

${ }^{5}$ Muzio fu l'unico allievo di Verdi, e presto divenne il suo assistente nella concertazione e nella messinscena. Nei primi anni cinquanta iniziò una carriera di direttore d'orchestra che, affiancata a una discreta notorietà come compositore, lo porterà in molti grandi teatri d'Europa e d'America. Verdi lo impiegava come consulente, mandandolo ad ascoltare cantanti ed esprimere giudizi; Muzio infatti fu anche maestro di canto, molto ricercato anche da artisti già in carriera (cfr. Gaspare Nello Vetro, L'allievo di Verdi Emanuele Muzio, Parma, Zara, 1993).
} 
puntò con decisione alla carriera teatrale, non disperdendo energie altrove, Frondoni iniziò la solita vita girovaga del musicista-artigiano, passando come maestro concertatore a Cagliari, Lodi, Genova e Milano. Così iniziò il suo rapporto con Bartolomeo Merelli, il leggendario impresario della Scala che nel decennio 1836-1845 portò a Milano Donizetti, Pacini, Mercadante e finalmente Verdi.

Quando nel 1838 Merelli vide la possibilità di mandare un maestro concertatore a Lisbona, che garantisse adeguate esecuzioni degli spartiti di sua proprietà, le ipotesi probabili furono due: Frondoni e Verdi appunto, o almeno così sembra. Sappiamo che Verdi proprio in quei mesi era senza impiego fisso, dopo le dimissioni da Busseto; sappiamo poi che Merelli lo guardava con deciso interesse; sappiamo infine che Verdi rifiutò una possibilità di lavoro a Monza. È quindi probabile che quando l'impresario del São Carlos di Lisbona António Lodi chiese a Merelli un giovane e affidabile maestro, aggiornato sulle nuove tendenze del melodramma italiano, venisse fuori anche il nome di Verdi ${ }^{6}$. Così come è verisimile che Verdi, ormai profondamente impegnato nella composizione della sua prima opera, abbia rifiutato uno spostamento che lo avrebbe completamente distratto. Frondoni, al contrario, era prima di tutto un direttore-concertatore, non un compositore, e deve aver colto l'occasione per una collocazione in un teatro lontano, ma prestigioso come quello della capitale portoghese. Già l'anno dopo, infatti, la

${ }^{6}$ La società musicale milanese conosceva le doti di concertatore di Verdi sin dal 1834, quando curò le prove e diresse l'esecuzione della Creazione di Haydn ai Filarmonici; l'episodio è stato raccontato innumerevoli volte; cfr. per esempio A. Oberdorfer, Giuseppe Verdi. Autobiografia dalle lettere, Milano, Rizzoli, 1951, pp. 57-58 (che cita le parole di Verdi stesso, dal cosiddetto "racconto autobiografico" del 1879, a Giulio Ricordi); F. Walker, Verdi l'uomo, Milano, Mursia, 1964, pp. 23-25; J. Black, "The Libretto of La Creazione del mondo. Milan, April 1834”, Studi Verdiani, 2, 1983, pp. 147-158; Mary Jane Phillips-Matz, Verdi. A Biography, Oxford-New York, Oxford Univ. Press, 1993, pp. 49-53. 
stampa lo indicava come principale responsabile della prima locale del Don Giovanni di Mozart, opera ancora considerata di difficile esecuzione ${ }^{7}$.

Sembrano due figure così lontane, Verdi e Frondoni, ma non dovettero sembrare tali a Merelli e ad António Lodi, poiché dal loro punto di vista i due mostravano più affinità di quante oggi potremmo immaginarne. Nel 1838 Verdi e Frondoni erano due giovani forestieri "immigrati” a Milano, promettenti e bisognosi di lavoro, gravitando intorno alla Scala e al suo impresario. La differenza semmai era un poco a favore di Frondoni, che aveva già portato in scena due titoli comici con discreta fortuna, mentre Verdi più giovane di quattro anni stava ancora faticando sul suo primo melodramma.

Alla fine del 1838 quindi Frondoni è ingaggiato dal conte Quintella di Farrobo a dirigere il São Carlos di Lisbona, dove rimarrà fino al 1843. Anche in questa nuova fase la sua vicenda non è così lontana da quella di Verdi. Per esempio nel 1844 Frondoni coglie il suo maggior successo con l'opera seria Os prófugos de Parga ispirato al poema di Giovanni Berchet (I profughi di Parga, 1819-1820), sul quale Francesco Hayez aveva realizzato uno dei suoi più celebri dipinti ${ }^{8}$. Le figure del profugo, dell'esule, del proscritto erano centrali nel discorso risorgimentale di quegli anni. Il tema quindi echeggiava molto da vicino le coeve opere verdiane, dall'Ernani al Corsaro, per non parlare del progetto mai realizzato di un Lorenzino de Medici, che occupò Verdi in questi anni 1844-

\footnotetext{
${ }^{7}$ Manuel Carlos de Brito, Luísa Cymbron, "Opera Orchestra in the 18th and 19th Centuries in Lisbon and Oporto", p. 458.

${ }^{8}$ Il dipinto risale al 1831, e segue di due anni il quadro forse più rivoluzionario di Hayez, I Lombardi alla Prima Crociata ispirato al poema di Tommaso Grossi. Quindi le due tele di Hayez, non per caso indicato da Mazzini come il pittore che meglio interpretava la funzione sociale del moderno artista, hanno ispirato rispettivamente Frondoni e Verdi; un ulteriore elemento in comune, pur dando per scontata la differenza di livello artistico.
} 
1846. Grazie a quel successo, Frondoni divenne una figura di spicco nel mondo teatrale portoghese e diresse anche il teatro Rua de Condes e il teatro Gymnasio. Convinto repubblicano, nel 1846 si avvicinò agli oppositori della casa regnante portoghese e compose l'inno Maria da Fonte per coro e orchestra, ancor oggi eseguito. Sorprende anche qui l'esatto parallelismo con quanto andava facendo Verdi, che il 21 aprile 1848, un mese dopo le Cinque Giornate di Milano, scrisse al suo librettista Francesco Maria Piave: "Ancora pochi anni forse pochi mesi e l'Italia sarà libera, una, repubblicana. Cosa dovrebbe essere?". E come Frondoni, anche Verdi nel 1849 compose un inno repubblicano, Suona la tromba, sui versi di Goffredo Mameli, che avrebbe dovuto diventare nelle intenzioni di Mazzini la "Marseillaise italiana".

Frondoni rappresenta quindi la più viva attualità musicale e politica italiana, esattamente nella linea del melodramma impegnato, militante, del Verdi coevo. La dirompente novità che essi rappresentano nel mondo operistico, quindi, consiste nella diversa relazione del melodramma con l'attualità, una novità che dall'Italia arriva fino al Portogallo, dove trova terreno fertile non solo dal punto di vista artistico. Non è quindi particolare che un repertorio o un direttore italiani si impongano nei teatri portoghesi. Ma lo è invece il fatto che la inedita temperatura politica dei compositori e delle loro opere si imponga tanto rapidamente anche fuori dei confini italiani. Eppure, solo nove anni prima dell'arrivo di Frondoni, Saverio Mercadante aveva composto per lo stesso São Carlos di Lisbona un Adriano in Siria sul celeberrimo, ma vecchissimo libretto di Metastasio. La distanza fra i due mondi è davvero abissale, e il radicale mutamento dell'orizzonte di attesa del pubblico portoghese, avvenuto in un solo decennio, offre la più chiara testimonianza delle condizioni in cui si sviluppò l'irresistibile ascesa del teatro di Verdi.

Su queste basi è possibile spiegare fenomeni apparentemente enigmatici in una società dove notizie, persone, tendenze e 
gusti artistici circolavano con maggior lentezza: l'esempio del Nabucco mi sembra significativo. Nel solo 1842 alla prima del 9 marzo seguirono 57 repliche, che occuparono la Scala fino agli ultimi mesi dell'anno. Pochi mesi dopo, il 29 ottobre 1843, l'opera arrivava già a Lisbona, e il 18 aprile 1844 a Porto. È davvero una rapidità sorprendente, se si pensa che il Nabucco arrivò solo nel 1844 a Vienna, capitale dell'impero di cui Milano stessa faceva parte, nel teatro imperiale gestito dallo stesso impresario Merelli. Nel 1838 Frondoni, nel 1843 Nabucco rappresentano due momenti chiave dell'espansione del nuovo melodramma italiano all'estero.

Ma se Frondoni fu sempre elogiato dalla stampa locale, non si può dire lo stesso per Verdi: una parte della critica giornalistica portoghese, quella conservatrice e legata alle concezioni rossiniane, reagì contro le novità del Nabucco: "non un'aria, non un duetto, non un pezzo completo; l'orchestra, mal diretta, [...] produce i peggiori effetti"'. Va riconosciuto tuttavia che la critica portoghese, esattamente come i migliori osservatori italiani, fra cui spicca il milanese Alberto Mazzucato, cambiarono immediatamente idea già a partire da Ernani. Anche quest'opera arrivò in Portogallo pochi mesi dopo la prima alla Fenice di Venezia: l'ambiente musicale locale condivise sin dalle prime esecuzioni la nuova drammaturgia dallo svolgimento impetuoso e la nuova temperatura del "gioco delle passioni" romantiche. Se anche i contenuti socio-politici legati alla storia d'Italia di quegli anni burrascosi dovettero essere scarsamente condivisi dal pubblico portoghese (certamente non incline a interpretare in senso simbolico attualizzante le storie del medioevo italico, o la storia romano-barbarica, o episodi di vita rinascimentale torbida e violenta), tuttavia non passò inosservato il nuovo impeto emozionale, la nuova rapidità d'azione, la

${ }^{9}$ Luísa Cymbron, "A produção e recepção das óperas de Verdi em Portugal no século XIX”, p. 24 (in trad.). 
concentrazione del tempo drammatico in attimi di altissima intensità, che caratterizzavano la generazione del Risorgimento. E infatti si forma in questi anni Quaranta anche in Portogallo un primo repertorio, un "canone verdiano", che rimarrà poi intatto fino a oggi. In esso entrano le opere che meglio testimoniano la mentalità della nuova generazione, e in particolare la concezione del tempo concentrato in attimi di sconvolgimento radicale, opposto ai tempi lunghi tanto del dramma classico quanto del melodramma di Bellini: Macbeth, Attila, I due Foscari e finalmente L'assedio di Arlem riscrittura librettistica della Battaglia di Legnano.

Con questi titoli siamo giunti a una nuova fase della vita di Verdi e della storia dell'opera italiana. È finita l'egemonia dei vecchi impresari Barbaja, Lanari e Merelli, ma l'esportazione di forza lavoro musicale dall'Italia non è affatto diminuita: quasi tutti i cantanti che eseguono le prime verdiane a Lisbona sono fra quelli che lo stesso mercato italiano considerava "di prima forza", e non è infrequente che nella prima locale fossero impegnati gli stessi cantanti della prima assoluta.

Quindi è vero che Verdi in persona non fu mai attivo in alcun teatro portoghese, è vero che non diresse mai personalmente, come fece a Vienna, Parigi, Londra, San Pietroburgo, ma è anche vero che, insieme al suo editore Ricordi, il compositore sorvegliava continuamente anche il mercato portoghese e tutelava le opere nuove garantendo esecutori fidati, preferibilmente già collaudati.

Il 28 febbraio 1855 cade la prima nazionale della Traviata al teatro di Porto (e il 29 ottobre viene ripresa a Lisbona). L'editore Ricordi manda per l'occasione una cantante fidatissima, particolarmente adeguata a rappresentare la figura emaciata ed estenuata della protagonista: Maria (Marietta) Spezia, che era stata Violetta nella fortunatissima première della versione riveduta al Teatro S. Benedetto di Venezia il 6 maggio 1854, dopo il fiasco dell'anno prima alla Fenice. La Spezia aveva creato uno stile nuovo, immediatamente com- 
preso ed esaltato dalla stampa italiana, era infatti cantante non di forza come la prima Violetta del 1853 (Fanny Salvini Donatelli, madre di Tommaso Salvini, il più celebre attore shakespeariano dell'Ottocento), ma dai mezzi vocali particolari, spesso quasi esangue, eccellente nella rappresentazione di un personaggio piegato dal dolore, schiacciato dal sacrificio, affaticato dalla malattia. Gli autori stessi, Verdi e Piave ${ }^{10}$, consideravano la Spezia l'interprete ideale per quel personaggio, proprio perché lontana dalla convenzione esecutiva del belcanto di tradizione e dalle pose impostate e improbabili dei cantanti di routine ${ }^{11}$. Sembra quindi molto significativo che la interprete in quel momento più richiesta come Violetta, certamente molto impegnata e sostenuta da Ricordi che la proponeva ai teatri maggiori di tutto il mondo, abbia tenuto le prime portoghesi proprio nel ruolo in cui Verdi stesso l'apprezzava sopra ogni altra ${ }^{12}$.

10 "Ho la soddisfazione di dirti che la Spezia è fatta per quest'opera, e che quest'opera pare fatta per la Spezia, e che se altro non succede e che i polmoni di questa eccellente giovinetta reggeranno, ella rappresenterà la nostra opera come nessuna al mondo potrà mai sognarsi di farlo. [...] In quest'opera è un'altra donna da quella di tutte le altre, e lo stesso pallore, e lo stesso sfinimento, e la persona tutta e la fisionomia, tutto concorre in lei a renderla la vera incarnazione del pensiero di Dumas, di Verdi, ed anche mio" (lettera di Piave a Verdi, 5 maggio 1854, in Franco Abbiati, Giuseppe Verdi, Milano, Ricordi, 1959, vol. 2, pp. 271-272).

11 "Voce debole, ma talento grande, anima e sentimento di scena" scrisse di lei Verdi l'11 novembre del 1856. E questo nuovo realismo della recitazione presto trova altre rappresentanti, soprattutto Maria Piccolomini e Virginia Boccabadati, affermandosi presto come lo stile dominante.

${ }^{12}$ Almeno in nota conviene ricordare che Verdi pensava alla Spezia come interprete ideale anche per il personaggio di Cordelia nel Re Lear, un progetto che andò avanti dal 1853 per lunghi anni, senza mai portare a nulla. I suoi ruoli verdiani furono Abigaille nel Nabucco, che cantò anche nella stagione 1855-56 del São Carlos, Amelia del Ballo in maschera, Leonora nel Trovatore, Gilda, Giselda nei Lombardi, Lucrezia nei Due Foscari, Luisa Miller: personaggi legati da caratteristiche psicologiche comuni, che evidentemente la Spezia interpretava con una distinta individualità. Non desta quindi meraviglia che per il personaggio di Cordelia fossero tenute in considerazione anche la Piccolomini e la Boccabadati, citate alla nota precedente, che avevano un repertorio simile, e dotate del nuovo stile esecutivo che Verdi immaginava. 
Il rinnovato e profondo favore che circondava la musica italiana in questo momento, contribuì in modo sostanziale a creare l'ambiente emozionale-affettivo in cui le sorti politiche d'Italia hanno trovato spazio nelle psicologie collettive europee. Senza ovviamente ipotizzare una relazione di causaeffetto, mi sembra chiaro tuttavia che tanto il processo verso l'unità, quanto il crescente favore per lo "spirito della nazione" italiana indotto nei pubblici europei dal melodramma verdiano, sono sintomi paralleli del clima di consenso in cui si è resa possibile l'unificazione italiana. Quindi se esiste un Verdi risorgimentale non è per precisi costrutti semantici, non per generici e retorici proclami ("Avrai tu l'universo, resti l'Italia a me"; "un soffio accendi, che dia morte allo straniero"; "Viva Italia! un sacro patto/tutti stringe i figli suoi" ecc.). Queste sono poco più che vuote parole, inutili ed eccessive se astratte dal momento storico in cui furono scritte, populismo pronto per la strumentalizzazione da Ventennio o per il patriottismo anacronistico e asinesco di alcuni padani attuali. Ciò che è importante, nell'affermazione del dramma verdiano, è la diffusione planetaria immediata di una nuova mentalità collettiva, una diversa percezione del tempo accelerato e in costante evoluzione, un favore per il dinamismo giovanile e vigoroso ma sostanziato non più soltanto di eroismo impulsivo, irresponsabile e muscoloso. Il nuovo spirito italiano che ora conquista l'Europa consiste soprattutto in una nuova disposizione psicologica collettiva, che va cercata anche nella musica di Verdi, nella sua immediatezza di comunicazione (nel senso di "non mediata" dalla riflessione), nella raffinatezza dei mezzi volontariamente semplificati per raggiungere una istantanea quanto profonda efficacia. E questo vale a qualsiasi latitudine, tanto a Manaus quanto a San Pietroburgo, tanto a L'Avana quanto a Lisbona, tanto a Buenos Aires quanto a Milano.

Un ballo in maschera, anche da questo punto di vista della psicologia collettiva internazionale, rappresenta un momento importante, e non stupisce quindi che arrivi al São Carlos 
solo dieci mesi dopo la prima romana, con una cantante di altissimo profilo come Marcellina Lotti Della Santa ${ }^{13}$ e con uno dai tenori preferiti da Verdi come Gaetano Fraschini ${ }^{14}$.

Se i commentatori portoghesi rilevarono subito la qualità di questa nuova musica "filosofica" di Verdi ${ }^{15}$, più o meno a partire dal Rigoletto e via via sempre più fino al secondo Simon Boccanegra, ciò non significa che abbiano decodificato i contenuti strettamente politici, legati agli eventi e alle fasi del processo risorgimentale italiano. "Filosofico" è il nuovo concetto di musica melodrammatica di Verdi, che ha sì la principale funzione di suscitare "impressioni, impressioni e nient'altro" ${ }^{16}$, ma che si discosta radicalmente dalla concezione kantiana della musica "piacevole", dilettevole "gioco di sensazioni”. Al contrario, la musica nel melodramma di Verdi è la componente che riempie di significati la vicenda, e sono questi significati, non la musica in sé, ciò che evidentemente i tempi nuovi volevano e sentivano. Per "significa-

${ }^{13}$ Marcellina Lotti della Santa (1831-1901) partecipò alla prima assoluta dell'Aroldo a Rimini nel 1857, sotto la direzione di Angelo Mariani, alla presenza di Verdi: una grande carriera internazionale. Aveva studiato con Alberto Mazzucato e il suo stile di canto era a mezza via fra la tradizione rossiniana di agilità e la nuova voce sombre più pesante e corposa. Forse proprio questa qualità la rese adeguata ad alcune figure del teatri di Verdi come Odabella nell'Attila, Amelia nel Ballo in maschera e Maria nel Boccanegra.

${ }^{14}$ Gaetano Fraschini (1816-1887), primo interprete dei Due Foscari, della Alzira, della più volte citata Battaglia di Legnano (nella parte del tenore martire Arrigo, che muore baciando la bandiera), del Trovatore e finalmente del ruolo di Gabriele Adorno nel Simon Boccanegra del 1857. Fraschini era celebrato anche per la qualità attoriale, e forse per questo tanto gradito a Verdi, che sempre assegnò grande rilievo alla visualità scenica.

${ }^{15}$ Luísa Cymbron, "A produção e recepção das óperas de Verdi em Portugal no século XIX”, p. 30.

${ }^{16}$ Lettera di Verdi a Cesare De Sanctis, 17 aprile 1872: "La musica è universale. Gli imbecilli ed i pedanti hanno voluto trovare ed inventare delle scuole, dei sistemi!!... Io vorrei che il pubblico giudicasse altamente, non colle miserabili viste dei giornalisti, maestri e suonatori di pianoforte, ma dalle sue impressioni! ... Capite? Impressioni, impressioni e niente altro?' (corsivi miei). 
ti”, ovviamente, non si intende qui qualcosa di semantizzabile, di traducibile in parole: questi significati sono invece da ricercare nelle strutture espressive, nella rapidità d'azione e nella sintesi espressiva delle pulsioni emotive, nella profondità di raffigurazione psicologica concentrata nelle poche battute della "mossa della melodia" (i memorabili inizi dei cantabili come "D'amor sull'ali rosee"; o delle cabalette come "Di quella pira"; o momenti melodici culminanti e brevissimi, che Verdi scrive attraverso l'intera vita, da "Già t'invoco, già ti sento,/dio verace d'Israello", a "Ah perché venni incauta", fino a "E son io l'innocente cagion di tanto pianto", dove l'evidente differenza di qualità poetica non influenza minimamente l'efficacia del "significato" melodico). È l'uomo nuovo, fatto di carne e nervi, con il sangue che gira più rapido nelle vene, con improvvise esaltazioni e irreparabili disperazioni, con nuove strutture mentali e una nuova psicologia per affrontare la vita, con il tempo accelerato della vita psichica devastata dallo scontro con la realtà, con l'instabilità e la fragilità di chi è esposto senza più sicurezze e protezioni al disordine degli eventi e dei cataclismi interiori (Rigoletto, Riccardo di Warwich, Otello, ma anche Violetta, Leonora, Eboli, Amneris ...).

Questo, ovviamente, non è un elemento distintivo del rapporto fra Verdi e il Portogallo, ma è su questi presupposti che può essere spiegata la rapidità con cui il melodramma verdiano intorno alla metà del secolo ha potuto diffondersi nel mondo intero in meno di dieci anni, dalla Russia zarista alla San Francisco del cercatori d'oro. I motivi per spiegare un tale fenomeno risiedono nelle strutture profonde della nuova sensibilità, nell'uomo che esce distrutto dalla fine dell'idealismo romantico: la fine delle certezze morali e spirituali, la fine della metafisica, la fine degli ideali alla Mazzini. Se c'è un senso del tragico nel medio Ottocento, un senso della fine irreparabile che annulla ogni possibilità d'azione dell'uomo, certo Verdi ne è uno dei massimi interpreti, col- 
locandosi in quella zona che, risvegliatasi dall'illusione mazziniana, non poteva certo accontentarsi delle certezze proposte dal positivismo né dalla nuova realpolitik nazionalista e imperialista.

Spetta agli storici della cultura portoghese indagare se questa situazione ha collegamenti dimostrabili con la storia della mentalità nazionale, della letteratura, della pittura e della filosofia portoghesi.

Dopo gli anni Settanta, quando ancora il direttore d'orchestra del São Carlos di Lisbona è il romano Raffaele Kuon ${ }^{17}$, l'egemonia italiana non termina, ma si intreccia con altre influenze, segnatamente quella francese e quella wagneriana. Ciò non impedisce che ancora a fine secolo lavorassero a Lisbona direttori italiani di grande notorietà come Marino Mancinelli e Cleofonte Campanini, due specialisti verdiani che hanno costantemente eseguito anche Wagner e i Francesi. Le prime locali del Don Carlos nel 1885, del secondo Boccanegra nel 1887, e dell'Otello nel 1889 sono affidate ancora a esecutori italiani. L'ultima, Otello, è diretta da Campanini e sua moglie Eva Tetrazzini è Desdemona, entrambi approvati da Verdi stesso nelle precedenti esecuzioni italiane e francesi.

Con ciò siamo alle soglie del Novecento e il canone è ormai fissato, predominando un criterio più ecumenico e sovranazionale, che comprende Verdi alla pari di Wagner, Gounod, Bizet, Massenet, Caikovskij ecc. Ciò non significa che sia finita una presunta "età dell'oro" verdiana, ma che quel coinvolgimento emotivo che aveva caratterizzato il medio Ottocento è stato sostituito da un atteggiamento storicistico

\footnotetext{
${ }^{17}$ Raffaele Kuon, appartenente a una famiglia di musicisti romani, per la precisione non fu un direttore d'opera ortodosso. La sua formazione con Tullio Ramacciotti, pioniere della rinascita strumentale romana, lo portò in contatto con Franz Liszt, allora residente nella città dei Papi. Presto tuttavia, forte dell'esperienza acquisita proprio nella musica d'insieme, iniziò la carriera di direttore; finirà la vita come strumentista girovago, a Cuneo, Per notizie rimando anche a Luísa Cymbron, "A produção e recepção das óperas de Verdi em Portugal no século XIX”, p. 37.
} 
per un "canone" museale. È insomma, in Portogallo come nel resto d'Europa, l'inizio di quell'atteggiamento che ha fatto del melodramma qualcosa di molto più simile a un pezzo da museo che a una raffigurazione emotivamente partecipata della vita contemporanea, ossia l'atteggiamento che predominerà attraverso il Novecento.

Se facciamo un salto di un secolo, sembra che la situazione sia quasi capovolta: i decenni che stiamo vivendo, fra XX e XXI secolo, hanno rinnovato quasi tutto nel sistema teatrale, dalla messinscena all'esecuzione vocale, dalla attorialità alla prassi strumentale, e grazie a ciò il melodramma vive una nuova contemporaneità grazie in primo luogo all'attività dei registi più avvertiti. La cosiddetta "regia critica", ormai pienamente accettata anche nel campo del melodramma, è una realtà, e tutti siamo abituati a regìe che sovrappongono al testo d'autore un'interpretazione attualizzante. Il bellissimo Falstaff che il São Carlos allestì nell'occasione del centenario verdiano del 2001, per fare un esempio, ha testimoniato quanto Verdi sia ancor oggi presente e necessario alla vita culturale del Portogallo, come nel resto del mondo. E, pur consapevole del potenziale negativo che si nasconde in ogni sentimento nazionalista, contro cui Reinhart Koselleck ha messo in guardia ${ }^{18}$, il prolungato rispetto e l'ammirazione universale per Verdi desta ancora oggi negli Italiani commozione e gratitudine. Se la reputazione italiana nel mondo è ai minimi storici, purtroppo con fondate ragioni, ancor oggi vale quanto quasi due secoli fa notava Heinrich Heine: quando si parla di melodramma, in molti degli Italiani soprattutto all'estero si accende ancora una luce di commozione e di vitalità, che riscatta da un fondo quasi di vergogna per le condizioni presenti dalla propria nazione, nel 1830 come nel 2013. Questo rende ancor più grave il compor-

${ }^{18}$ Reinhart Koselleck, Il vocabolario della modernità, Bologna, Il Mulino, 2009 (ediz. orig. 2006), pp. 127-129. 
tamento dei governi italiani, che con una coerenza e sistematicità senza flessioni da almeno trent'anni si impegnano a distruggere la vita teatrale nazionale, costretta ad emigrare per sopravvivere.

Evidentemente, per qualcuno, questa è globalizzazione! 